\title{
氧化-温控双功能离子液体催化 $\mathrm{O}_{2}$ 氧化模拟柴油脱硫

\author{
于凤丽 ${ }^{a}$ 唐会宝 $^{a}$ 柳春玉 $^{a}$ 解从霞* ${ }^{a}$ 于世涛 $^{b}$ \\ ( ${ }^{a}$ 青岛科技大学化学与分子工程学院 青岛 266042) \\ ( ${ }^{b}$ 青岛科技大学化工学院 青岛 266042)
}

\begin{abstract}
摘要 将温控结构单元聚醚链和催化氧化基团磷铇酸根共同引入到离子液体的结构中, 合成出一系列不同聚合度的十 八胺类氧化-温控双功能离子液体, 并将其应用于催化 $\mathrm{O}_{2}$ 氧化模拟柴油的脱硫反应过程, 实现了反应分离一体化, 并 取得了良好的脱硫效果和催化剂循环使用效果. 该类离子液体在甲苯/正十二烷混合溶剂中具有良好的温控性能. 篮选 出活性较高的离子液体催化剂, 其聚合度 $n=111$ 以及烷基碳链为 $\mathrm{C}_{12}$. 考察了反应温度、反应时间和氧气压力等因素 对脱硫效果的影响. 在优化的反应条件 ( $V$ (甲苯) $: V$ (模拟油) $\left.=1: 1, T=100{ }^{\circ} \mathrm{C}, t=2 \mathrm{~h}, p\left(\mathrm{O}_{2}\right)=2.0 \mathrm{MPa}\right)$ 下, $\mathrm{DMF}$ 萃取一 次, 脱硫率接近 100\%. 催化剂循环使用 11 次, 脱硫率仍可达到 $95 \%$ 以上.
\end{abstract}

关键词 功能化离子液体; 温控; 氧化脱硫; 氧气

\section{Oxidative Desulfurization of Model Diesel with $\mathrm{O}_{2}$ Catalyzed by Oxidative-thermoregulated Bifunctional Ionic Liquids}

\author{
Yu, Fengli $^{a} \quad$ Tang, Huibao $^{a} \quad$ Liu, Chunyu $^{a} \quad$ Xie, Congxia ${ }^{* a} \quad$ Yu, Shitao ${ }^{b}$ \\ ( ${ }^{a}$ College of Chemistry and Molecular Engineering, Qingdao University of Science and Technology, Qingdao 266042) \\ ( ${ }^{b}$ College of Chemical Engineering, Qingdao University of Science and Technology, Qingdao 266042)
}

\begin{abstract}
A series of ammonium oxidative-thermoregulated bifunctional ionic liquids (ILs) have been synthesized by introducing the thermoregulated structural unit (PEG chain) into ammonium cation and using heteropolyanion as catalytic oxidation group. The prepared ionic liquids have the thermoregulated solubility in toluene/ $n$-dodecane mixed solvent. IL/toluene/ $n$-dodecane system owns the feature of "homogeneous at high temperature, heterogeneous at low temperature". The effects of the dosage of the ionic liquid, the polymerization degree of the ionic liquid, and the volume ratio of toluene to $n$-dodecane on the thermoregulation of the system have been investigated. Model diesel is prepared by dissolving the model sulfur-containing compound thiophene in $n$-dodecane. By using the ionic liquid as catalyst, the thermoregulated IL/toluene/ $n$-dodecane biphasic system has been used for catalytic oxidative desulfurization of model diesel with $\mathrm{O}_{2}$ as oxidant to achieve the integration of homogeneous reaction and heterogeneous separation. So, the highly efficient catalysis and the simply separation of catalyst are achieved. The selected catalytic system is the polymerization degree of the ionic liquid $n$ $=111$ and $V$ (toluene) $: V($ model oil $)=1: 1$. The effects of the alkyl chain of the ionic liquid, reaction temperature, reaction time and the pressure of oxygen on the desulfurization rate have been fully investigated. The selected optimal conditions are as follows: the alkyl chain $\mathrm{C}_{12}$, reaction temperature $100{ }^{\circ} \mathrm{C}$, reaction time $2 \mathrm{~h}$, and the pressure of oxygen $2.0 \mathrm{MPa}$. Under the optimal conditions, the sulfur in model diesel nearly can be completely removed by using DMF as extraction agent. After the reaction, the catalyst can be automatically precipitated from the oil phase at the room temperature. Therefore, the recycle of the catalyst is easily realized. The recyclability of the ionic liquid catalyst is investigated. The result has showed that the catalyst exhibits very excellent recyclability. Under the above selected reaction conditions, the desulfurization rate still can reach above $95 \%$ after 11 recycles of the ionic liquid catalyst.

Keywords task-specific ionic liquids; thermoregulation; oxidative desulfurization; oxygen
\end{abstract}

\section{1 引言}

随着全球范围内机动车需求的不断增大, 机动车尾 气排放已成为大气污染的主要来源之一. 燃油中的硫 醇、硫醚和噻吩等有机硫化物在燃烧过程中释放的 $\mathrm{SO}_{X}$
可形成酸雨、导致大气污染. 因此，世界各国环境法规 对燃油的硫质量分数上限要求越来越低, 发达国家对燃 油含硫量的要求尤高. 为满足环保要求并提高我国油品 的国际竞争力, 开展燃油深度脱硫技术的研究, 已成为 炼油工业的当务之急 ${ }^{[1,2]}$.

\footnotetext{
*E-mail: xiecongxia@126.com

Received August 6, 2014; published October 22, 2014.

Project supported by the National Natural Science Foundation of China (No. 21176129), the Foundation for Outstanding Young Scientist in Shandong Province (No. BS2011NJ008) and the Project of Basic Research in Qingdao City (No. 13-1-4-194-jch).

项目受国家自然科学基金(No. 21176129)、山东省优秀中青年科学家科研奖励基金(No. BS2011NJ008)和青岛市科技发展计划项目(No. 13-1-4-194-jch) 资助.
} 
传统的催化加氢脱硫技术很难实现深度脱硫, 因为 噻吩类等硫化物的加氢活性低, 很难被完全脱除 ${ }^{[3 \sim 5]}$. 另外, 催化加氢脱硫的反应条件苛刻、设备投资和操作 费用高、氢气消耗量大. 在目前已发展的多种非加氢脱 硫技术中，催化氧化脱硫技术具有明显的优越性 ${ }^{[6 ~ 9]}$. 催化氧化脱硫是利用氧化剂在催化剂作用下将油品中 的硫化物氧化成极性大的砜、亚砜，再利用燃油与砜、 亚砜极性差距大的特点, 通过选择合适的有机萃取剂分 离出砜、亚砜, 从而达到脱硫的目的. 可见, 催化氧化脱 硫的操作条件相对温和、设备投资较低, 并且更容易脱 除在加氢脱硫中难以去除的苯并噻吩和二苯并噻吩类 硫化物. 因此, 催化氧化脱硫更易实现深度脱硫.

在目前已有的催化氧化脱硫研究中, 以过氧化氢做 氧化剂的研究最为成熟 ${ }^{[10 ~ 14]}$. 反应可以在较为温和的 条件下进行, 但这需要消耗大量的过氧化氢, 成本也较 高. 由于以 $\mathrm{O}_{2}$ 或空气为氧化剂不仅能够克服 $\mathrm{H}_{2} \mathrm{O}_{2}$ 受热 易分解等固有的缺点, 而且价廉易得, 被认为是最有发 展前途的环境友好型氧化剂. 催化 $\mathrm{O}_{2}$ 氧化脱硫的研究 已有文献报道 ${ }^{[15 ~ 20]}$. 杂多酸及其盐可用作均相或多相

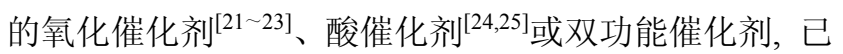
引起了人们广泛的关注, 其用于催化氧化脱硫, 表现出 较高的催化活性 ${ }^{[26 ~ 29]}$. 磷铇酸活化氧气性能在 1996 年 已有文献报道 ${ }^{[21]}$, 基于磷铇酸的离子液体在脱硫反应 中的应用也有文献报道 ${ }^{[27]}$, 其脱硫反应活化氧气的机 理和文献类似 ${ }^{[17,18]}$.

最近, 课题组利用离子液体的可设计性, 将温控结 构单元聚醚链和催化氧化基团磷铇酸根共同引入到离 子液体的结构中, 合成出一系列不同聚合度的十八胺类 氧化-温控双功能离子液体 $\mathbf{1}$, 并将其应用于催化 $\mathrm{H}_{2} \mathrm{O}_{2}$ 氧化模拟燃油的脱硫反应过程 ${ }^{[30]}$. 利用离子液体的温 控性能, 实现了脱硫过程的反应分离一体化; 利用离子 液体的催化氧化性能, 催化 $\mathrm{H}_{2} \mathrm{O}_{2}$ 氧化模拟柴油脱硫, 脱 硫率为 $93 \%$. 本文用 $\mathrm{O}_{2}$ 代替 $\mathrm{H}_{2} \mathrm{O}_{2}$, 以 1 为催化剂, 用于 模拟柴油的催化氧化脱硫. 不仅可以实现脱硫过程的反 应分离一体化, 而且获得了良好的脱硫效果. 在优化的 反应条件下, 脱硫率可接近 100\%. 另外, 催化剂 1 也呈 现出良好的循环使用能力.

$$
\begin{aligned}
& \left.\left[\mathrm{R}_{8} \underset{\mathrm{R}}{\mathrm{N}}-\left(\mathrm{CH}_{2} \mathrm{CH}_{2} \mathrm{O}\right)_{\mathrm{a}} \mathrm{H} \mathrm{CH}_{2} \mathrm{O}\right)_{\mathrm{b}} \mathrm{H}\right]_{3} \mathrm{PW}_{12} \mathrm{O}_{40}^{3-} \\
& \mathrm{R}=n-\mathrm{C}_{4} \mathrm{H}_{9}, n-\mathrm{C}_{8} \mathrm{H}_{17}, n-\mathrm{C}_{12} \mathrm{H}_{25}
\end{aligned}
$$

1

\section{2 结果与讨论}

\section{1 离子液体的温控性能}

具有 “高温均相, 低温分相” 特性的有机液/液两相 温控体系一般由极性、非极性及其弱极性溶剂组成, 并 且极性与非极性溶剂不互溶, 弱极性溶剂与非极性和极
性溶剂都互溶 ${ }^{[31,32]}$. 选择正十二烷和甲苯分别作为非极 性和弱极性溶剂, 研究了极性的离子液体在甲苯/正十 二烷混合溶剂中的温控性能. 研究结果表明, 离子液 体、正十二烷和甲苯组成的体系具有 “高温均相, 低温 分相” 的特性, 以离子液体为催化剂, 可以实现温控相 分离催化.

固定甲苯的用量, 在甲苯和正十二烷的体积比为 $1: 1$ 的体系中, 考察了烷基链长为 $\mathrm{C}_{8}$ 离子液体 $(\mathrm{R}=$ $\left.n-\mathrm{C}_{8} \mathrm{H}_{17}\right)$ 的用量和聚合度 $(n=\mathrm{a}+\mathrm{b})$ 对温控性能的影响, 结果如表 1 所示. 由表 1 可知, 相同聚合度的离子液体, 随着离子液体用量的减少, 其临界溶解温度升高; 不同 聚合度的离子液体，随着聚合度的增加，在相同温度下 实现均相时, 其用量减少. 这是因为离子液体在甲苯中 具有最低溶解度, 和文献[30]的研究结果是一致的.

表 1 离子液体在甲苯和正十二烷体积比为 $1: 1$ 体系下的温控性能 Table 1 Thermoregulation of ionic liquids in $1: 1$ volume ratio of toluene to $n$-dodecane

\begin{tabular}{cccccccc}
\hline \multirow{2}{*}{$\begin{array}{c}\text { 聚合度 } \\
n\end{array}$} & $m(\mathrm{IL}): m$ (甲苯) & \multicolumn{7}{c}{ 温度 $/{ }^{\circ} \mathrm{C}$} \\
\cline { 3 - 8 } 63 & 3.9495 & 65 & 70 & 75 & 80 & 85 & 90 \\
\hline \multirow{2}{*}{63} & 2.1516 & $\mathrm{i}$ & $\mathrm{i}$ & $\mathrm{i}$ & $\mathrm{ps}$ & $\mathrm{s}$ & - \\
& 1.9208 & $\mathrm{i}$ & $\mathrm{i}$ & $\mathrm{i}$ & $\mathrm{i}$ & $\mathrm{ps}$ & $\mathrm{s}$ \\
& 3.8310 & $\mathrm{i}$ & $\mathrm{i}$ & $\mathrm{ps}$ & $\mathrm{s}$ & - & - \\
& 2.8314 & $\mathrm{i}$ & $\mathrm{i}$ & $\mathrm{i}$ & $\mathrm{ps}$ & $\mathrm{s}$ & - \\
& 1.8186 & $\mathrm{i}$ & $\mathrm{i}$ & $\mathrm{i}$ & $\mathrm{i}$ & $\mathrm{ps}$ & $\mathrm{s}$ \\
& 2.0263 & $\mathrm{i}$ & $\mathrm{i}$ & $\mathrm{ps}$ & $\mathrm{s}$ & - & - \\
& 1.7700 & $\mathrm{i}$ & $\mathrm{i}$ & $\mathrm{i}$ & $\mathrm{ps}$ & $\mathrm{s}$ & - \\
& 1.5467 & $\mathrm{i}$ & $\mathrm{i}$ & $\mathrm{i}$ & $\mathrm{i}$ & $\mathrm{ps}$ & $\mathrm{s}$ \\
\hline
\end{tabular}

${ }^{a}$ Insoluble; ${ }^{b}$ partly soluble; ${ }^{c}$ soluble.

另外，考察了不同甲苯和正十二烷体积比的体系， 在同一温度 $\left(90{ }^{\circ} \mathrm{C}\right)$ 下实现均相时离子液体的最少用量, 结果如表 2 所示. 由表 2 可知, 随着正十二烷体积比的 增加，离子液体的用量也增加. 综合考虑，体系用于催 化脱硫反应时模拟油品的用量不宜太少, 即正十二烷的 比例不宜太小; 另一方面, 离子液体作为催化剂其用量 不宜太多. 因此, 选定聚合度 $n=111$ 的离子液体 $(n=$ $\left.3.65 \times 10^{-5} \mathrm{~mol}\right), V($ 甲苯 $): V($ 正十二烷 $)=1: 1$ 的温控 体系用于催化脱硫反应.

表 2 离子液体 $\left[n=111\left(\mathrm{C}_{8}\right)\right.$ ]在不同甲苯和正十二烷体积比体系下的 温控性能

Table 2 Thermoregulation of the ionic liquid $\left[n=111\left(\mathrm{C}_{8}\right)\right]$ in different volume ratio of toluene to $n$-dodecane

$V$ (甲苯) $: V$ (正十二烷) $\quad 1: 1 \quad 1: 1.1 \quad 1: 1.2 \quad 1: 1.3 \quad 1: 1.4 \quad 1: 1.5$

$m(\mathrm{IL}): m$ (甲苯) $\quad \begin{array}{lllllll}1.5467 & 2.0171 & 2.8724 & 4.9866 & 5.7268 & 7.500\end{array}$

\section{2 离子液体的烷基链长对脱硫率的影响}

将硫化物噻吩溶于正十二烷中配成含硫的模拟柴 油. 在甲苯和模拟油品的体积比为 $1: 1$ 的体系中, 将不 
同烷基链长 $(\mathrm{R})$ 的离子液体 $\mathbf{1}(n=111)$ 用于催化模拟燃 油的 $\mathrm{O}_{2}$ 氧化脱硫，结果如表 3 所示.

表 3 离子液体的烷基链长对脱硫率的影响 ${ }^{a}$

Table 3 Effect of carbon chain in ionic liquid on the desulfurization rate

\begin{tabular}{clll}
\hline 烷基链长 & $\mathrm{C}_{4}$ & $\mathrm{C}_{8}$ & $\mathrm{C}_{12}$ \\
\hline 脱硫率 $/ \%$ & 93.9 & 95.2 & 99.8 \\
\hline$a$ 反应条件: $\mathrm{O}_{2}$ 压力 $2.0 \mathrm{MPa}$ & 反应泹度 $100{ }^{\circ} \mathrm{C}$ 反应时间 $2 \mathrm{~h}$ &
\end{tabular}

由表 3 可知, 随着烷基链长的增加, 脱硫率增加. 其中, 碳链为 $\mathrm{C}_{12}$ 的离子液体催化效果最佳, 脱硫率接 近 100\% (DMF 萃取 1 次). 烷基链长(R)对离子液体的温 控性能影响不大, 虽然在选择的条件下, 三种离子液体 在反应温度下都能实现均相催化, 但是碳链为 $\mathrm{C}_{12}$ 的离 子液体显示出最高的催化活性, 这有可能是因为其与以 正十二烷为组分的模拟燃油的相溶性最好的原因. 因 此, 选择碳链为 $\mathrm{C}_{12}$ 的离子液体为催化剂, 进行反应条 件的优化.

\section{3 反应温度对脱硫率的影响}

以离子液体 $n=111\left(\mathrm{C}_{12}\right)$ 为催化剂, 在甲苯和模拟 油品的体积比为 $1: 1$ 、氧气压力为 $2.0 \mathrm{MPa}$ 、反应时间 为 $2 \mathrm{~h}$ 的条件下, 考察了反应温度对脱硫率的影响, 结 果如图 1 所示.

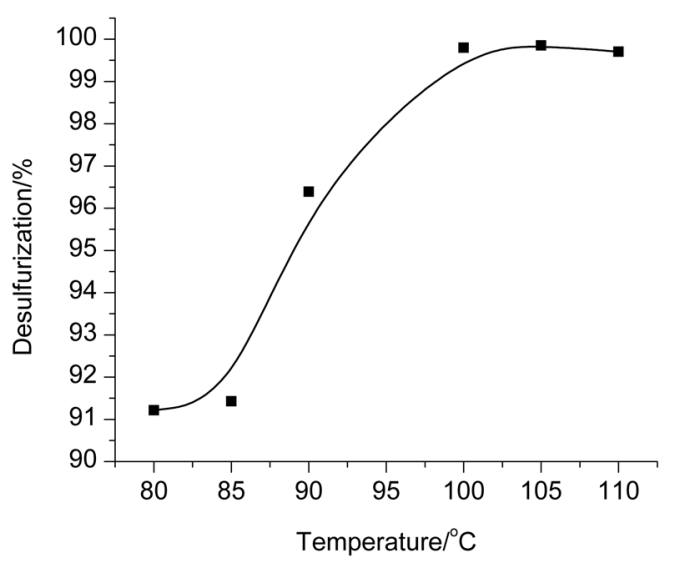

图 1 反应温度对脱硫率的影响

Figure 1 Effect of reaction temperature on the desulfurization rate

由图 1 可知, 在临界溶解温度 $\left(90{ }^{\circ} \mathrm{C}\right)$ 以下反应时, 脱硫率较低, 而在临界溶解温度 $\left(90{ }^{\circ} \mathrm{C}\right)$ 以上反应时, 由 于体系为均相, 催化剂活性高, 因此脱硫率明显升高. 在 $100{ }^{\circ} \mathrm{C}$ 反应时, 脱硫率接近 $100 \%$, 进一步升高反应 温度, 脱硫率变化不大. 因此, 最佳的反应温度为 100 ${ }^{\circ} \mathrm{C}$.

\section{4 反应时间对脱硫率的影响}

以离子液体 $n=111\left(\mathrm{C}_{12}\right)$ 为催化剂, 在甲苯和模拟 油品的体积比为 $1: 1$ 、氧气压力为 $2.0 \mathrm{MPa}$ 、反应温度 为 $100{ }^{\circ} \mathrm{C}$ 的条件下, 考察了反应时间对脱硫率的影响, 结果如图 2 所示.

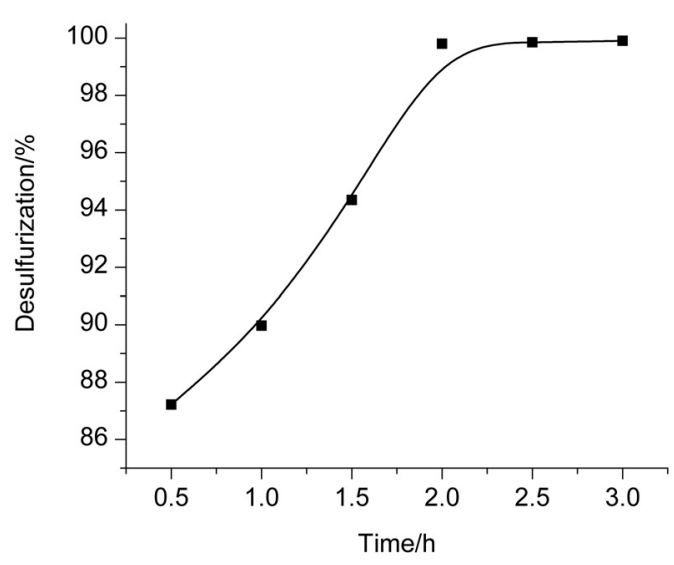

图 2 反应时间对脱硫率的影响

Figure 2 Effect of reaction time on the desulfurization rate

由图 2 可知，随着反应时间的延长，脱硫率增加， 当反应时间达到 $2 \mathrm{~h}$ 时, 脱硫率接近 $100 \%$, 进一步延长 反应时间, 脱硫率变化不大. 因此, 最佳的反应时间为 $2 \mathrm{~h}$.

\section{5 氧气压力对脱硫率的影响}

以离子液体 $n=111\left(\mathrm{C}_{12}\right)$ 为催化剂, 在甲苯和模拟 油品的体积比为 $1: 1$ 、反应温度为 $100{ }^{\circ} \mathrm{C}$ 、反应时间 为 $2 \mathrm{~h}$ 的条件下, 考察了氧气压力对脱硫率的影响, 结 果如图 3 所示.

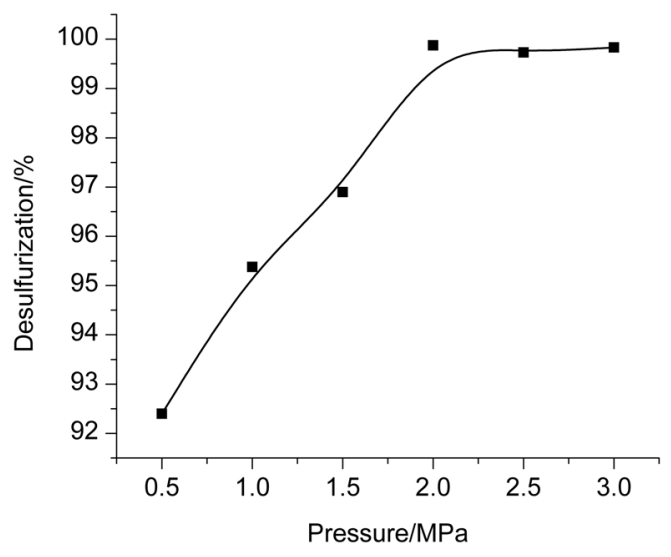

图 3 氧气压力对脱硫率的影响

Figure 3 Effect of oxygen pressure on the desulfurization rate

由图 3 可知, 在氧气压力低于 $2.0 \mathrm{MPa}$ 时, 脱硫率 随着氧气压力的不断增加而增大; 当氧气压力达到 2.0 $\mathrm{MPa}$ 时, 脱硫率接近 $100 \%$, 继续增加氧气压力, 脱硫率 无明显变化. 由此可见, 氧气的最佳压力为 $2.0 \mathrm{MPa}$.

总之, 在以上选择的最佳条件下，催化剂 1 催化 $\mathrm{O}_{2}$ 氧化模拟柴油脱硫，脱硫率可接近 $100 \%$; 而催化剂 1 催 化 $\mathrm{H}_{2} \mathrm{O}_{2}$ 氧化模拟柴油脱硫, 在最佳的条件下脱硫率仅 为 $93 \%{ }^{[30]}$. 由此可见, 由于以 $\mathrm{O}_{2}$ 为氧化剂能够克服 $\mathrm{H}_{2} \mathrm{O}_{2}$ 本身受热易分解等固有的缺点, 因此催化剂 $\mathbf{1}$ 催化 
$\mathrm{O}_{2}$ 比催化 $\mathrm{H}_{2} \mathrm{O}_{2}$ 的活性高、脱硫效果好.

另外, 需要说明的是: 本实验的研究对象是以正十 二烷为主要成分的模拟油品, 而实际油品中本身就含有 一定比例的芳香烃(主要以甲苯为主), 因此建立的温控 体系如果应用于实际油品, 并不需要使用大量的甲苯, 这也正是选择甲苯为弱极性溶剂的初衷所在; 另外, 也 可以通过调节离子液体的用量来实现温控而不需要使 用大量的甲苯.

\section{6 催化剂的循环利用效果}

利用催化剂的温控性能, 可以实现反应分离一体 化. 反应结束后, 降至室温, 催化剂自动从反应体系中 析出, 分离出上层甲苯和油品相, 下层的催化剂可直接 循环使用. 在甲苯和模拟油品的体积比为 $1: 1$ 、氧气压 力为 $2.0 \mathrm{MPa}$ 、反应温度为 $100{ }^{\circ} \mathrm{C}$ 和反应时间为 $2 \mathrm{~h}$ 的 条件下, 考察了离子液体催化剂 $n=111\left(\mathrm{C}_{12}\right)$ 的循环使 用效果，结果如图 4 所示.

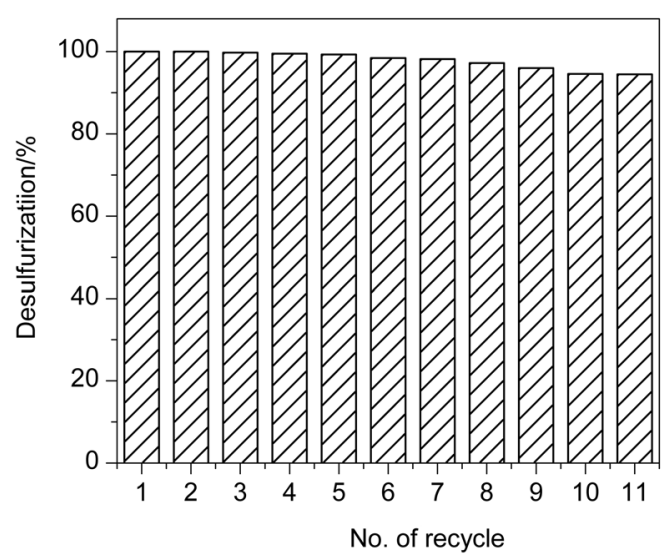

图 4 催化剂的循环使用效果

Figure 4 Recycling efficiency of the catalyst

由图 4 可知, 催化剂具有良好的循环使用效果. 催 化剂循环使用 5 次, 脱硫率仍接近 100\%; 循环使用 11 次，脱硫率仍可达到 $95 \%$ 以上.

\section{3 结论}

十八胺类氧化-温控双功能离子液体用于催化 $\mathrm{O}_{2}$ 氧 化模拟柴油的脱硫反应过程, 实现了反应分离一体化, 并取得了良好的脱硫效果和催化剂循环使用效果. 篮选 出活性最高的离子液体催化剂, 其聚合度 $n=111$ 以及 烷基碳链为 $\mathrm{C}_{12}$. 在优化的反应条件 ( $V$ (甲苯) : $V$ (模拟 油 $\left.)=1: 1, T=100{ }^{\circ} \mathrm{C}, t=2 \mathrm{~h}, p\left(\mathrm{O}_{2}\right)=2.0 \mathrm{MPa}\right)$ 下, DMF 萃取一次, 脱硫率接近 $100 \%$. 催化剂循环使用 11 次, 脱硫率仍可达到 $95 \%$ 以上. 本文为实现深度脱硫提供了 一种环境友好的新途径.

\section{4 实验部分}

离子液体 1 参考文献[30]合成. 将噻吩溶于正十二
烷中配成含硫量为 $700 \mu \mathrm{g} / \mathrm{mL}$ 的模拟柴油. 向 $100 \mathrm{~mL}$ 高压釜中依次加入 $3.65 \times 10^{-5} \mathrm{~mol}$ 催化剂 $1,5 \mathrm{~mL}$ 模拟 柴油和 $5 \mathrm{~mL}$ 甲苯, 放入转子, 封釜. 检验气密性, 用 $\mathrm{O}_{2}$ 置换釜内空气 4 次, 然后充入一定压力的 $\mathrm{O}_{2}$. 开启搅拌, 在油浴中一定温度下反应一定时间. 待高压釜完全冷却 后，开釜，将高压釜内混合物取出置于 $25 \mathrm{~mL}$ 比色管中， 静置 $20 \mathrm{~min}$, 取上层清液用等体积的 DMF $\left(40{ }^{\circ} \mathrm{C}\right.$ 水浴 中摚拌 $10 \mathrm{~min}$ )萃取一次. 分相后, 取上层清液用 WK-2D 型微库仑综合分析仪测定脱硫率. 对氧化产物 进行 GC-FPD 检测, 结果表明氧化产物主要为噻吩砜. 比色管中下层催化剂可重新加入高压釜循环使用.

\section{References}

[1] Kulkarni, P. S.; Afonso, C. A. M. Green Chem. 2010, 12, 1139.

[2] Zhao, D. S.; Li, J. P.; Zhang, J.; Ren, P. B.; Ge, J. J.; Ren, T. J.; Cui, Y. Chin. J. Org. Chem. 2014, 37, 1462. (赵地顺, 李俊盼, 张娟, 任培兵, 葛京京, 任腾杰, 崔云, 有机化学, 2014, 37, 1462.)

[3] Rothlisberger, A.; Prins, R. J. Catal. 2005, 235, 229.

[4] Bej, S. K.; Maity, S. K.; Turaga, U. T. Energy Fuels 2004, 18, 1227.

[5] Liu, W. Q.; Zhou, H.; Lei, W. N.; Shang, T. M.; Zhang, Q.; Sun, K. D. Acta Chim. Sinica 2011, 69, 1622. (刘维桥, 周虎，雷卫宁，尚 通明, 张强, 孙桂大, 化学学报, 2011, 69, 1622.)

[6] Campos-Martin, J. M.; Capel-Sanchez, M. C.; Fierro, J. L. G. Green Chem. 2004, 6, 557.

[7] Lo, W. H.; Yang, H. Y.; Wei, G. T. Green Chem. 2003, 5, 639.

[8] Zhang, W.; Xu, K.; Zhang, Q.; Liu, D. L.; Wu, S. Y.; Verpoort, F.; Song, X. M. Ind. Eng. Chem. Res. 2010, 49, 11760.

[9] Zhu, W. S.; Wu, P. W.; Yang, L.; Chang, Y. H.; Chao, Y. H.; Li, H. M.; Jiang, Y. Q.; Jiang, W.; Xun, S. H. Chem. Eng. J. 2013, 229, 250 .

[10] Zhang, W.; Xu, K.; Zhang, Q.; Liu, D. L.; Wu, S. Y.; Verpoort, F.; Song, X. M. Ind. Eng. Chem. Res. 2010, 49, 11760.

[11] Jiang, W.; Zhu, W. S.; Chang, Y. H.; Li, H. M.; Chao, Y. H.; Xiong, J.; Liu, H.; Zhu, F. X. Energy Fuels 2014, 28, 2754.

[12] Xu, J. H.; Zhao, S.; Chen, W.; Wang, M.; Song, Y. F. Chem. Eur. J. 2012, 18, 4775

[13] Lu, L.; Cheng, S. F.; Gao, J. B.; Gao, G. H.; He, M. Y. Energy Fuels 2007, 21, 383.

[14] Zhao, D. S. H.; Wang, J. L.; Zhou, E. P. Green Chem. 2007, 9, 1219.

[15] Rao, T. V.; Sain, B.; Kafola, S.; Nautiyal, B. R.; Sharma, Y. K.; Nanoti, S. M.; Garg, M. O. Energy Fuels 2007, 21, 3420.

[16] Zhou, X. R.; Li, J.; Wang, X. N.; Jin, K.; Ma, W. Fuel Process Technol. 2009, 90, 317

[17] Lu, H.; Zhang, Y. Y. N.; Jiang, Z. X.; Li, C. Green Chem. 2010, 12, 1954.

[18] Tang, N. F.; Zhang, Y. N.; Lin, F.; Lu, H. Y.; Jiang, Z. X.; Li, C. Chem. Commun. 2012, 48, 11647.

[19] Lü, H. Y.; Gao, J. B.; Jiang, Z. X.; Yang, Y. X.; Song, B.; Li, C. Chem. Commun. 2007, 6, 150.

[20] Tang, N. F.; Zhao, X. P.; Jiang, Z. X.; Li, C. Chin. J. Catal. 2014 35, 1433. (唐南方, 赵小平, 蒋宗轩, 李灿, 催化学报, 2014, 35, 1433.)

[21] Hill, C. L.; Damica Gall, R. J. Mol. Catal. A: Chem. 1996, 114, 103.

[22] Wang, S. S.; Liu, W.; Wan, Q. X.; Liu, Y. Green Chem. 2009, 11, 1589.

[23] Qiao, Y. X.; Hou, Z. S.; Li, H.; Hu, Y.; Feng, B.; Wang, X. R.; Hua, L.; Huang, Q. F. Green Chem. 2009, 11, 1955.

[24] Yang, H. G.; Qi, X. F.; Wen, L.; Lu, C. X.; Cheng, G. B. Ind. Eng. Chem. Res. 2011, 50, 11440.

[25] Leng, Y.; Wang, J.; Zhu, D. R.; Ren, X. Q.; Ge, H. Q.; Shen, L. Angew. Chem., Int. Ed. 2009, 48, 168.

[26] Zhu, W. S.; Wu, P. W.; Chao, Y. H.; Li, H. M.; Zou, F.; Xun, S. H.; Zhu, F. X.; Zhao, Z. Ind. Eng. Chem. Res. 2013, 52, 17399.

[27] Huang, W. L.; Zhu, W. S.; Li, H. M.; Shi, H.; Zhu, G. P.; Liu, H.; Chen, G. Y. Ind. Eng. Chem. Res. 2010, 49, 8998

[28] Yu, F. L.; Wang, R. Acta Chim. Sinica 2014, 72, 105. (于凤丽, 王 点, 化学学报, 2014, 72, 105.)

[29] An, Y.; Lu, L.; Li, C. M.; Cheng, S. F.; Gao, G. H. Chin. J. Catal. 
2009, 30, 1222. (安荣, 陆亮, 李才猛, 程时富, 高国华, 催化学 报, 2009, 30, 1222.)

[30] Yu, F. L.; Wang, Y. Y.; Liu, C. Y.; Xie, C. X.; Yu, S. T. Chem. Eng. J. 2014, 255, 372 .
[31] Griee, I. D.; Hvarey, P. J.; Jenkins, I. D. Tetrahedron Lett. 1996, 37, 1087.

[32] Behr, A.; Miao, Q. J. Mol. Catal. A: Chem. 2004, 222, 127.

(Zhao, X.) 